\title{
Optical Bistability in a Controllable Giant Self-Kerr Nonlinear Gaseous Medium under Electromagnetically Induced Transparency and Doppler Broadening
}

\author{
Phuong Le Thi Minh, ${ }^{1,2}$ Doai Le Van, ${ }^{1}$ Khoa Dinh Xuan, ${ }^{1}$ and Bang Nguyen Huy $\mathbb{D}^{1}$ \\ ${ }^{1}$ Vinh University, Le Duan 182, Vinh City, Vietnam \\ ${ }^{2}$ Saigon University, 273 An Duong Vuong, Ho Chi Minh City, Vietnam \\ Correspondence should be addressed to Bang Nguyen Huy; bangnh@vinhuni.edu.vn
}

Received 5 January 2018; Accepted 26 February 2018; Published 15 April 2018

Academic Editor: Jiayang Wu

Copyright (C) 2018 Phuong Le Thi Minh et al. This is an open access article distributed under the Creative Commons Attribution License, which permits unrestricted use, distribution, and reproduction in any medium, provided the original work is properly cited.

\begin{abstract}
We study optical bistability $(\mathrm{OB})$ in a controllable giant self-Kerr nonlinear atomic gaseous medium placed in a unidirectional ring cavity. The medium is coherently excited by strong controlling field and a weak probe laser field under electromagnetically induced transparency (EIT) and Doppler broadening. In a weak field limit of the probe light, an analytic OB equation for the probe light field is derived as an analytic function of parameters of the controlling field and temperature of the medium. It is shown that OB characters can be manipulated with the parameters due to the controllable properties of the self-Kerr nonlinearity. Furthermore, enhancement of the Kerr nonlinearity reduces the switching intensity threshold and width of the OB.
\end{abstract}

\section{Introduction}

The $\mathrm{OB}$ is one of the most interesting fields of research in nonlinear optics because it has a large number of potential applications in both optical sciences and photonic technology, for example, all-optical switches, all-optical memories, optical transistors, and all-optical logic gates [1-3]. For the OB systems using Kerr nonlinear materials, a large nonlinearity is a fundamental need to gain sensitivity. In the early years of the $\mathrm{OB}$ research for atomic media, a great interest was focused on the two-level atomic system due to resonantly enhanced nonlinearity $[1,2]$. However, applications of the two-level atomic system are limited due to strong resonant absorption and only one optical field being employed for both applying and switching, thus lacking control for switching intensity thresholds.

The advent of EIT [4] offers coherent media of vanished resonant absorption [5-8] and controllable giant Kerr nonlinearity [9-11]. Wang et al. performed a direct measurement of self-Kerr nonlinear coefficient in a three-level lambda EIT medium in the presence of Doppler broadening by using a cavity scanning technique. The measurement shows a great enhancement of the Kerr nonlinear index of refraction in several orders of magnitude around atomic resonance [12]. Recently, Doai et al. [13] have developed an analytical model of EIT enhanced self-Kerr nonlinearity with controllable parameters of a light field and temperature of atomic sample which is in good agreement with the experimental observations [12]. The studies showed that the Kerr nonlinearity is greatly enhanced and depends on temperature of the medium. Furthermore, the magnitude, slop, and sign of the Kerr nonlinear coefficient can be controlled by tuning frequency and/or intensity of the coupling light or by changing temperature of the medium. Such controllable giant Kerr nonlinear media are used for controllable optical bistability [14], generating four-wave mixing beams [15] which exploit new ways in designing devices for optical switching in optical communication and all-optical signal processing.

Therefore, owing to the controllable giant-nonlinearity of the EIT medium, switching intensity thresholds and width of the OB can be manipulated. For the first time, Joshi et al. [14] demonstrated experimentally the controllable atomic optical 
bistability in the three-level EIT system inside an optical ring cavity in the presence of Doppler broadening. Since then, aspects that influence OB hysteresis loop have been extensively investigated [16-26]. Similar optical bistability and vacuum Rabi splitting of ring cavity in EIT medium are well known theoretically and experimentally [27-29]. Several reviews [1,2] and monographs [3] on this field of the OB are available, which give a deeper insight into the topic and provide lists of original references.

In the $\mathrm{OB}$ studies related to the EIT atomic gaseous medium, it can be categorized into two types of OB which concern the dispersive and absorptive properties of the medium, respectively [3]. The dispersive OB type arises from the linear and nonlinear contributions, among which the giant Kerr nonlinearity often dominates because it is enhanced by atomic coherence $[12,13]$. However, study of $\mathrm{OB}$ concerning Kerr nonlinearity and temperature of such medium is still quite modest. Furthermore, in several cases, a precise knowledge of the relationship of the input and output intensities as a function of the controlling light and temperature of the medium is crucially needed to optimize the operating processes and to choose experimental parameters. With the growth of this interest, in this work, we studied controlling $\mathrm{OB}$ of the three-level EIT atomic gaseous medium under presence of giant self-Kerr nonlinearity and Doppler broadening by using an analytic method. Using the weak field approximation of the probe light, a relationship of output and input intensities of $\mathrm{OB}$ is derived in the presence of Doppler broadening. The influences of Doppler broadening and the controlling light on switching intensity thresholds and width of the OB are investigated.

\section{The Mater Equation of $\mathbf{O B}$}

We consider an atomic medium (with length $L$ ) composed of three-level $\Lambda$-type atoms with density $N$ placed in unidirectional ring cavity, as shown in Figure 1(a). A weak probe laser field with frequency $\omega_{p}$ and electric field amplitude $E_{p}$ drives the transition $|1\rangle \leftrightarrow|2\rangle$, whereas an intense coupling laser field with frequency $\omega_{c}$ and electric field amplitude $E_{c}$ couples a transition between the states $|2\rangle$ and $|3\rangle$.

For simplicity, we assumed both mirrors 1 and 2 are perfect reflectors; each has a reflectivity $R$ and transmittivity $T$, with $R+T=1$. In the unidirectional ring cavity, a part of the probe field $E_{p}$ is circulated in the cavity but not the coupling field $E_{c}$. The coherent field $E_{p}^{I}$ enters through mirror $M_{1}$, interacts with the atomic medium of the length $L$, circulates in the cavity, and partially comes out of the mirror $M_{2}$ as $E_{p}^{T}$. Part of the output intensity reflected back into the medium provides the feedback which is essential for generating $\mathrm{OB}$.

The relationship of the output and input intensities of the unidirectional ring cavity or master equation of $\mathrm{OB}$ due to the self-Kerr nonlinearity is given by [30]:

$$
I_{\text {out }}=\left[\frac{1}{2}+\frac{1}{2} \cos \left(\frac{2 \pi L}{\lambda} n_{2} I_{\text {out }}+\varphi\right)\right] I_{\text {in }} \text {, }
$$

where $n_{2}$ is the Kerr coefficient, $\lambda$ is wavelength of the probe light, $I_{\text {in }} \propto\left(E_{p}^{I}\right)^{2}$ and $I_{\text {out }} \propto\left(E_{p}^{T}\right)^{2}$ are incident and transmitted intensities of the probe light, and

$$
\varphi=\frac{2 \pi L}{\lambda} n_{0}+\varphi_{0}
$$

is the round-trip phase of the probe light, $\varphi_{0}$ is the initial phase, and $n_{0}$ is the linear index of refraction.

The self-Kerr coefficient $n_{2}$ can be determined by using the density matrix formalism. Under the framework of the semiclassical theory and dipole and rotating wave approximations, the evolution of the three-level system can be represented by [13]

$$
\begin{aligned}
\dot{\rho}_{11}= & \gamma_{31}\left(\rho_{33}-\rho_{11}\right)+\gamma_{21} \rho_{22}-\frac{i}{2} \Omega_{p} \rho_{21}+\frac{i}{2} \Omega_{p} \rho_{12}, \\
\dot{\rho}_{22}= & -\left(\gamma_{23}+\gamma_{21}\right) \rho_{22}-\frac{i}{2} \Omega_{p} \rho_{12}+\frac{i}{2} \Omega_{p} \rho_{21}-\frac{i}{2} \Omega_{c} \rho_{32} \\
& +\frac{i}{2} \Omega_{c} \rho_{23}, \\
\dot{\rho}_{33}= & \gamma_{31}\left(\rho_{11}-\rho_{33}\right)+\gamma_{23} \rho_{22}+\frac{i}{2} \Omega_{c} \rho_{32}-\frac{i}{2} \Omega_{c} \rho_{23}, \\
\dot{\rho}_{21}= & -\left(\gamma-i \Delta \rho_{p}\right)+\frac{i}{2} \Omega_{p}\left(\rho_{22}-\rho_{11}\right)-\frac{i}{2} \Omega_{c} \rho_{31}, \\
\dot{\rho}_{23}= & -\left(\gamma-i \Delta_{c}\right) \rho_{23}+\frac{i}{2} \Omega_{c}\left(\rho_{22}-\rho_{33}\right)-\frac{i}{2} \Omega_{p} \rho_{13}, \\
\dot{\rho}_{31}= & -\left[\gamma_{31}-i\left(\Delta \Delta_{p}-\Delta_{c}\right)\right] \rho_{31}+\frac{i}{2} \Omega_{p} \rho_{32}-\frac{i}{2} \Omega_{c} \rho_{21},
\end{aligned}
$$

where

$$
\begin{gathered}
\Delta_{p}=\omega_{p}-\omega_{21}, \\
\Delta_{c}=\omega_{c}-\omega_{23},
\end{gathered}
$$

is frequency detunings of the probe coupling lasers, respectively; $\Omega_{p}=d_{21} E_{p} / \hbar$ and $\Omega_{c}=d_{23} E_{c} / \hbar$ are the Rabi frequencies associated with the probe and coupling beams, respectively; $d_{l m}$ represents the dipole moment of the $|l\rangle-|m\rangle$ transition; $\gamma_{l m}$ is represented with the decay rates $\Gamma_{l m}$ from $|l\rangle$ to $|m\rangle$ as follows [13]:

$$
\begin{gathered}
\gamma_{l m}=\frac{1}{2}\left(\sum_{k<l} \Gamma_{l k}+\sum_{j<m} \Gamma_{m j}\right), \\
\gamma=\frac{\gamma_{21}+\gamma_{31}+\gamma_{23}}{2} .
\end{gathered}
$$

In (3), the diagonal matrix elements $\rho_{m m}(m=1 \div 3)$ represent population in the state $|m\rangle$, whereas the off-diagonal matrix elements $\rho_{l m}(l \neq m)$ represent the coherence between the states $|l\rangle$ and $|m\rangle$. Using perturbation theory, the matrix element $\rho_{21}$ is calculated up to third order as [13]

$$
\begin{aligned}
\rho_{21}= & \frac{-i \Omega_{p}}{2 F}\left(1-\frac{2 \gamma_{31}}{2 \gamma+\gamma_{21}}\right) \\
& +\frac{i \Omega_{p}}{2 F} \frac{\Omega_{p}^{2}}{2 \gamma+\gamma_{21}}\left(\frac{1}{F}+\frac{1}{F^{*}}\right),
\end{aligned}
$$




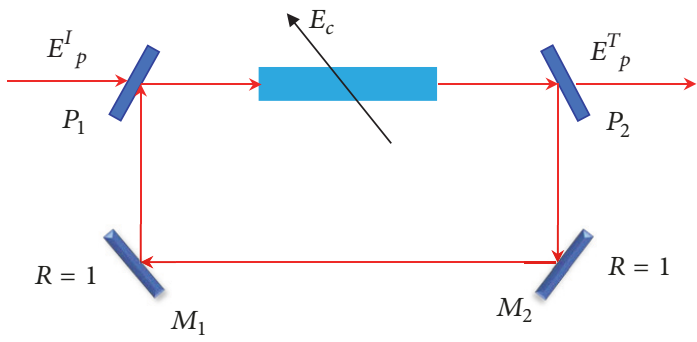

(a)

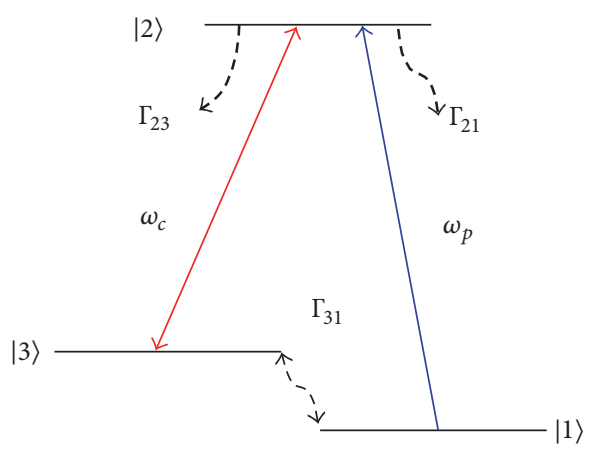

(b)

FIGURE 1: Schematic setup of a unidirectional ring cavity (a) containing a three-level atomic sample (b), where $E_{p}^{I}$ and $E_{p}^{T}$ denote the incident and transmitted probe field, respectively, and $E_{c}$ represents the coupling field that is not circulated inside the cavity.

where

$$
F=\gamma-i \Delta_{p}+\frac{\Omega_{c}^{2} / 4}{\gamma_{31}-i\left(\Delta_{p}-\Delta_{c}\right)},
$$

and $F^{*}$ is the complex conjugation of $F$.

For matrix element $\rho_{21}$, the total susceptibility $\chi$ for the probe light is determined by

$$
\chi=-2 \frac{N d_{21}}{\varepsilon_{0} E_{p}} \rho_{21} .
$$

In alternative form, the total susceptibility $\chi$ can be represented with the first-order and the third-order susceptibilities $\left(\chi^{(1)}\right.$ and $\left.\chi^{(3)}\right)$ by the following way:

$$
\chi=\chi^{(1)}+3 E_{p}^{2} \chi^{(3)}
$$

For a gaseous phase, it is necessary to take into account Doppler broadening. In such case, the linear and third-order nonlinear susceptibilities can be derived as [13]

$$
\begin{aligned}
\chi^{(1)} & =\frac{i N_{0} d_{21}^{2} \sqrt{\pi}}{\varepsilon_{0} \hbar\left(\omega_{p} u / c\right)}\left(1-\frac{2 \gamma_{31}}{2 \gamma+\gamma_{21}}\right) e^{z^{2}}[1-\operatorname{erf}(z)], \\
\chi^{(3)} & =-\frac{i N_{0} d_{21}^{4}}{3 \sqrt{\pi} \varepsilon_{0} \hbar^{3}\left(\omega_{p} u / c\right)^{2}}\left(\frac{1}{2 \gamma+\gamma_{21}}\right) \\
& \times\left\{2 \sqrt{\pi}\left(-1+\sqrt{\pi} z e^{z^{2}}[1-\operatorname{erf}(z)]\right)\right. \\
& \left.+\frac{\pi\left(e^{z^{2}}[1-\operatorname{erf}(z)]+e^{z^{* 2}}\left[1-\operatorname{erf}\left(z^{*}\right)\right]\right)}{z+z^{*}}\right\},
\end{aligned}
$$

where $u=\sqrt{2 k_{B} T / m}$ is the root mean square velocity, $N_{0}$ is the total atomic density of the vapor,

$$
z=\frac{c}{\omega_{p} u}\left(\gamma-i \Delta_{p}+\frac{\Omega_{c}^{2} / 4}{\gamma_{31}-i\left(\Delta_{p}-\Delta_{c}\right)}\right)=\frac{c}{\omega_{p} u} F,
$$

$z^{*}$ is the complex conjugation of $z$, and erf is the error function.

Having the linear and third-order nonlinear susceptibilities, a nonlinear Kerr index of refraction $n_{2}$ is determined by the following relations:

$$
n_{2}=\frac{3 \operatorname{Re}\left(\chi^{(3)}\right)}{4 \varepsilon_{0} n_{0}^{2} c},
$$

where $n_{0}=\sqrt{1+\operatorname{Re}\left(\chi^{(1)}\right)}$ is the linear index of refraction.

The self-Kerr nonlinear coefficient given be (12) represents a function of the intensity and frequency detuning of the coupling light and temperature of the medium. By substituting expression (12) into master equation (1) the $\mathrm{OB}$ behaviors can be investigated with the parameters.

\section{Analysis of $\mathbf{O B}$}

In order to analyze the analytic result of $\mathrm{OB}$, we consider the ${ }^{87} \mathrm{Rb}$ atomic gaseous medium. In this case, the states $|1\rangle,|2\rangle$, and $|3\rangle$ are chosen as $5 S_{1 / 2}(F=1), 5 P_{1 / 2}(F=2)$, and $5 S_{1 / 2}$ $(F=2)$, respectively. We used values for the parameters as follows (in which the frequencies and decay rates are given in the unit of $2 \pi)[12,31]: \lambda=780 \times 10^{-9} \mathrm{~m}, \omega_{p}=377 \times 10^{12} \mathrm{~Hz}$, $\gamma_{21}=3 \mathrm{MHz}, \gamma_{31}=1.1 \mathrm{MHz}, \gamma=3.5 \mathrm{MHz}$, and $N=4.5 \times$ $10^{17}$ atoms $/ \mathrm{m}^{3}, d_{21}=1.6 \times 10^{-29} \mathrm{C} \cdot \mathrm{m}$.

In the first step, we plotted a surface of $\mathrm{OB}$ at $\Delta_{c}=0, \Omega_{c}=$ $70 \mathrm{MHz}$ and $T=300 \mathrm{~K}$ and plotted $\mathrm{OB}$ curves at particular values of the probe frequency, as shown in Figures 2(a) and 2(b). It is shown that the threshold intensity and width of $\mathrm{OB}$ decrease as the probe frequency moves to the blue side. To explain this case, we plotted the Kerr nonlinear coefficient $n_{2}$ with the respect to the probe frequency detuning $\Delta_{p}$ (Figure 2(c)).

Indeed, the value of $n_{2}$ is given at $\Delta_{p}=-10 \mathrm{MHz}$, $-15 \mathrm{MHz}$, and $-20 \mathrm{MHz}$ is $7.2 \times 10^{-6} \mathrm{~cm}^{2} / \mathrm{W}, 5.1 \times 10^{-6} \mathrm{~cm}^{2} / \mathrm{W}$, and $3.2 \times 10^{-6} \mathrm{~cm}^{2} / \mathrm{W}$, respectively. The results show that, in the region of $\Delta_{p}$ from $-20 \mathrm{MHz}$ to $10 \mathrm{MHz}$, amplitude of the Kerr nonlinearity increases as frequency of the probe field moves to the blue and thus lowers the OB threshold 


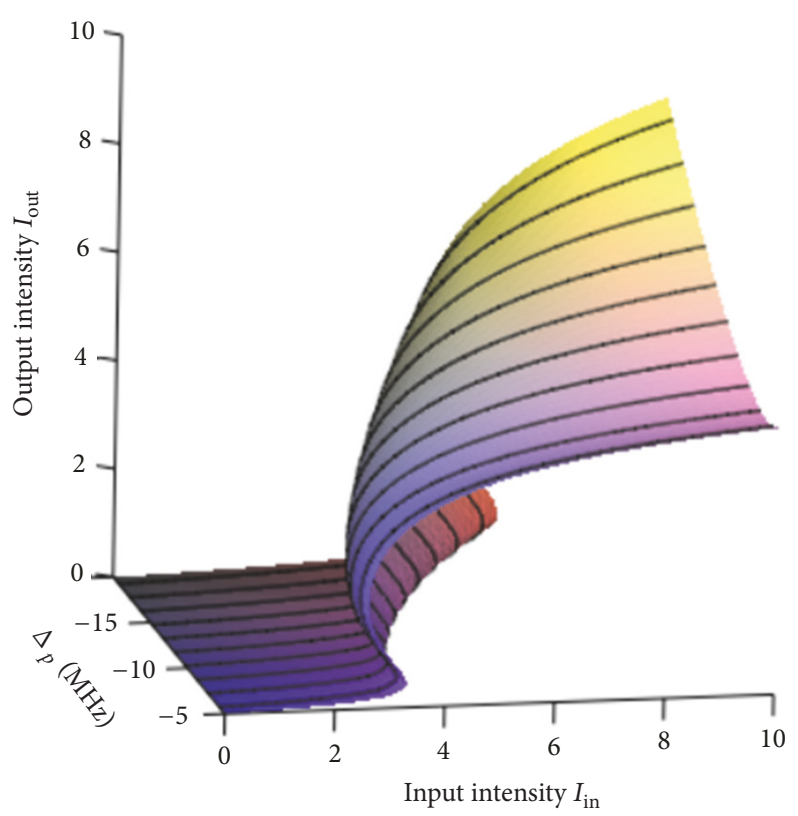

(a)

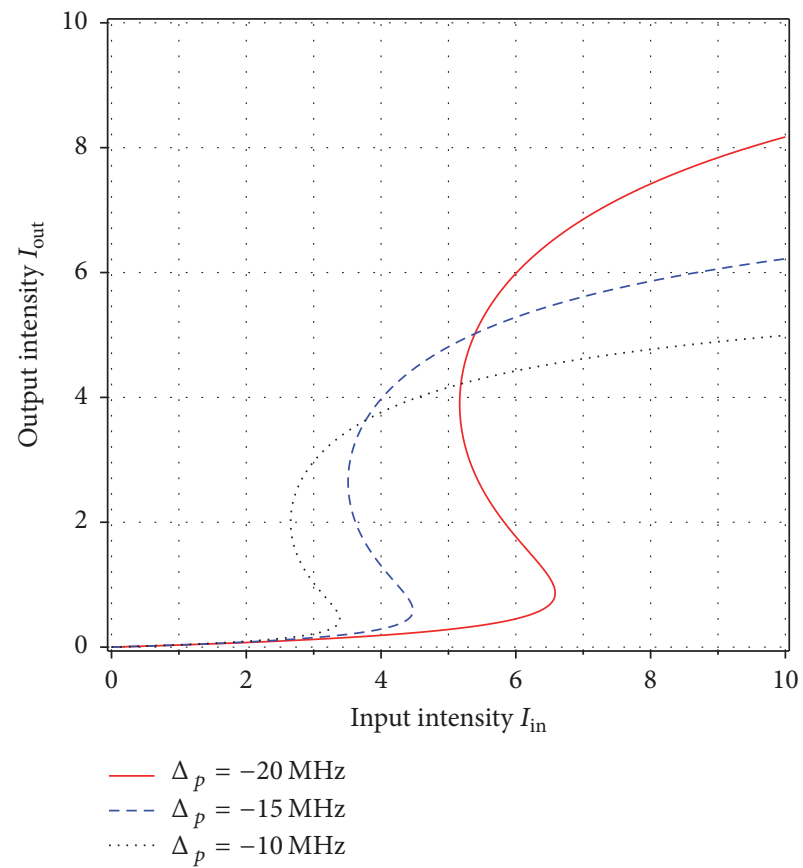

(b)

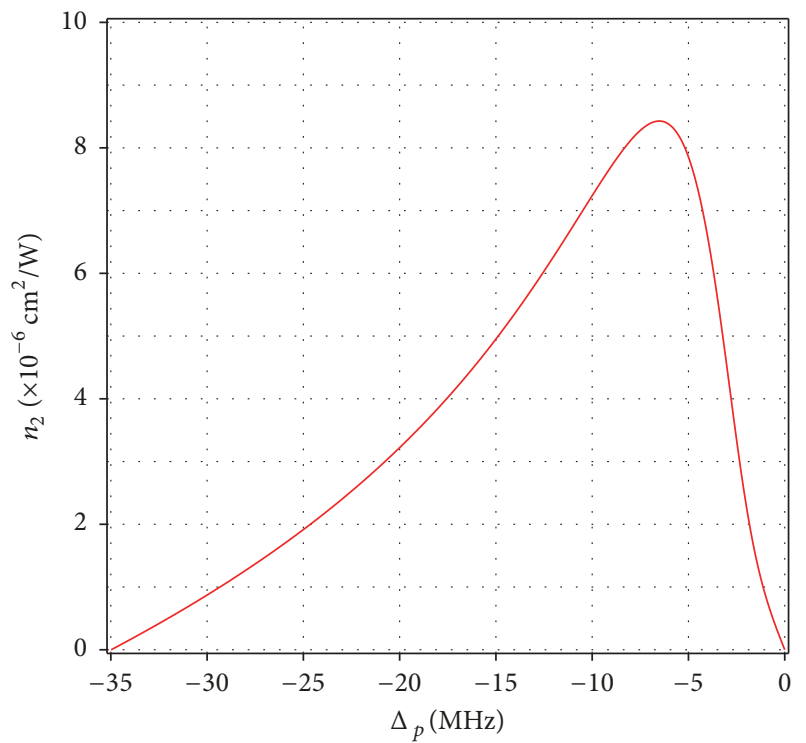

(c)

FIGURE 2: (a) Surface plot of the OB; (b) OB curves at particular values of the probe frequency detuning; (c) variation of the Kerr nonlinear coefficient $n_{2}$ versus the probe detuning. All the plots given at the fixed values $\Delta_{c}=0, \Omega_{c}=70 \mathrm{MHz}$, and $T=300 \mathrm{~K}$.

and $\mathrm{OB}$ width. Furthermore, for the given parameters of the coupling light and temperature of the medium, one can find a frequency region from $\Delta_{p}=-35 \mathrm{MHz}$ to $0 \mathrm{MHz}$ with positive value of the self-Kerr nonlinearity which can be used for OB (see Figure 2(c)). It is worth emphasizing that the magnitude of self-Kerr coefficient here reaches order $10^{-5} \mathrm{~cm}^{2} / \mathrm{W}$ that is much larger than that of the conventional Kerr materials [26].
Next, we investigated influences of the frequency and intensity of the coupling field by making plots of $O B$ curves at particular values of these parameters, as shown in Figures 3 and 4, respectively. In the first case, the threshold and width of the $\mathrm{OB}$ change with variation of frequency of the coupling field. This can be attributed (see Figure 3(b)) to changing the Kerr nonlinearity. Indeed, the values of $n_{2}$ at $\Delta_{c}=3 \mathrm{MHz}$, $7 \mathrm{MHz}$, and $15 \mathrm{MHz}$ are determined as $3.7 \times 10^{-6} \mathrm{~cm}^{2} / \mathrm{W}$, 


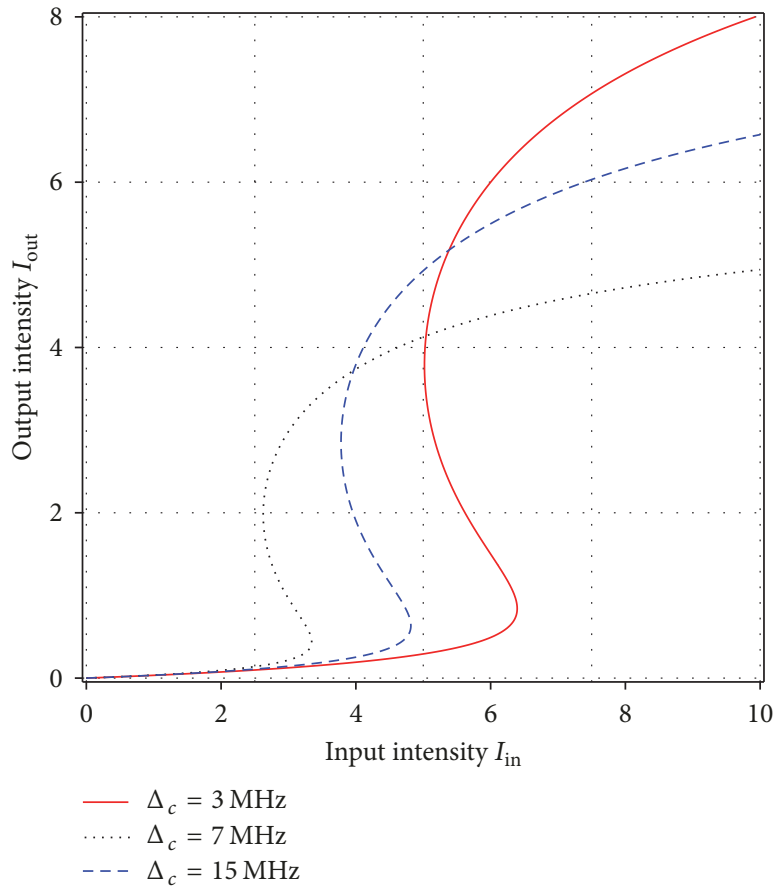

(a)

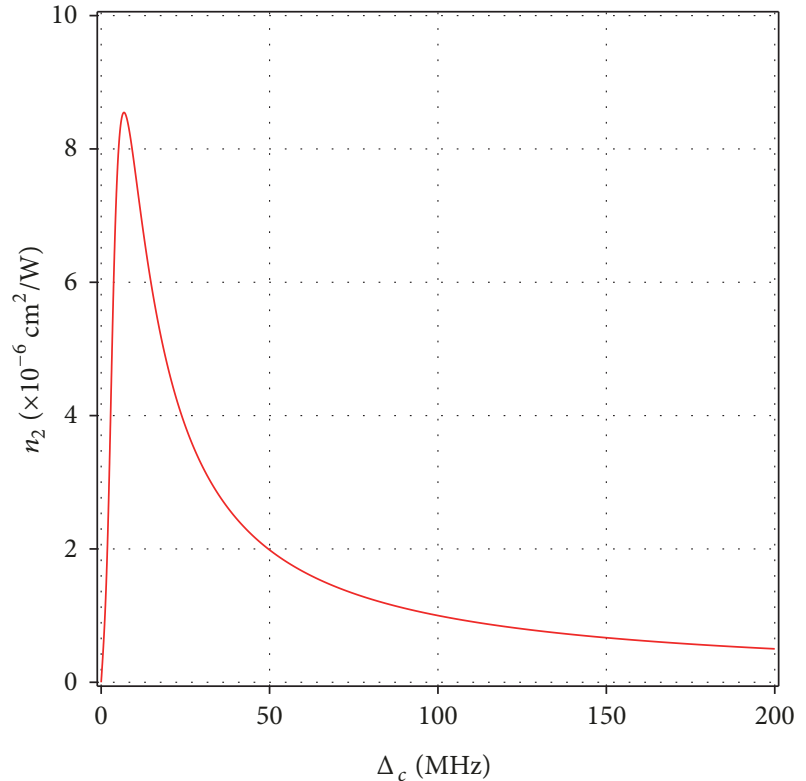

(b)

FIGURE 3: OB curves at particular values of frequency detuning of the coupling field (a) and variation of Kerr coefficient $n_{2}$ versus the coupling detuning (b). The parameters are $\Omega_{c}=70 \mathrm{MHz}, \Delta_{p}=0$, and $T=300 \mathrm{~K}$.

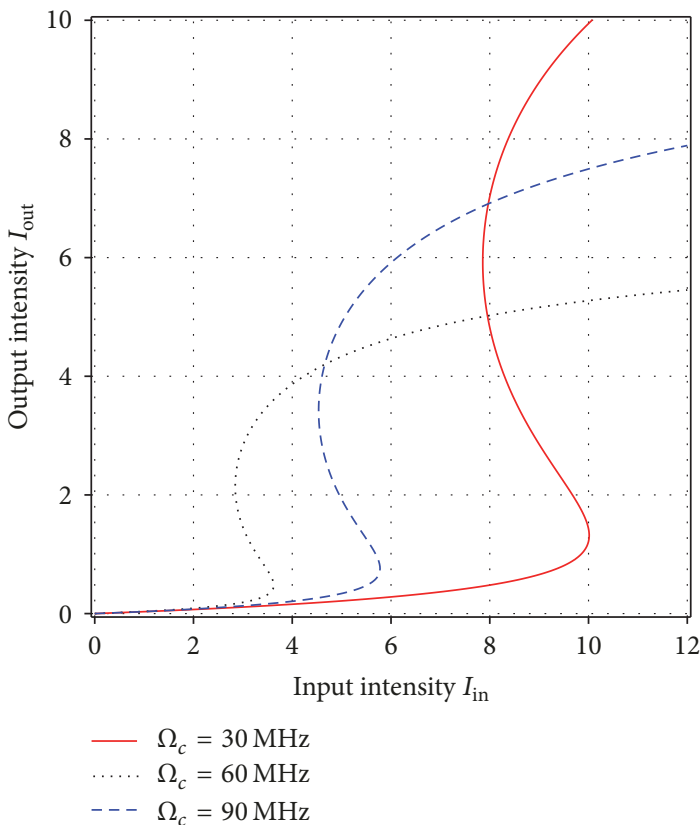

(a)

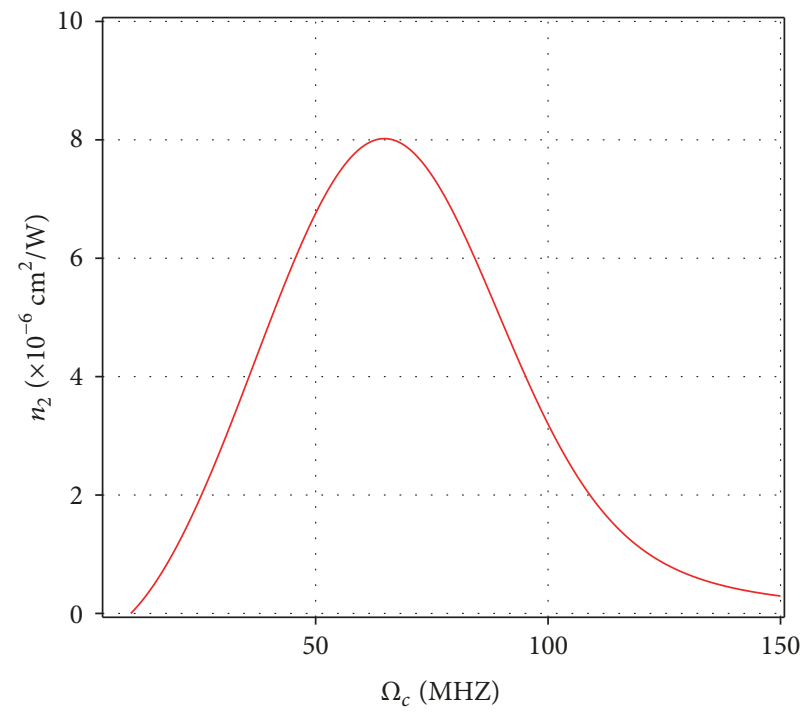

(b)

FIGURE 4: OB curves at particular values of intensity of the coupling field (a) and variation of Kerr coefficient $n_{2}$ versus intensity of the coupling field (b). The parameters are $\Delta_{p}=-5 \mathrm{MHz}, \Delta_{c}=0$, and $T=300 \mathrm{~K}$. 


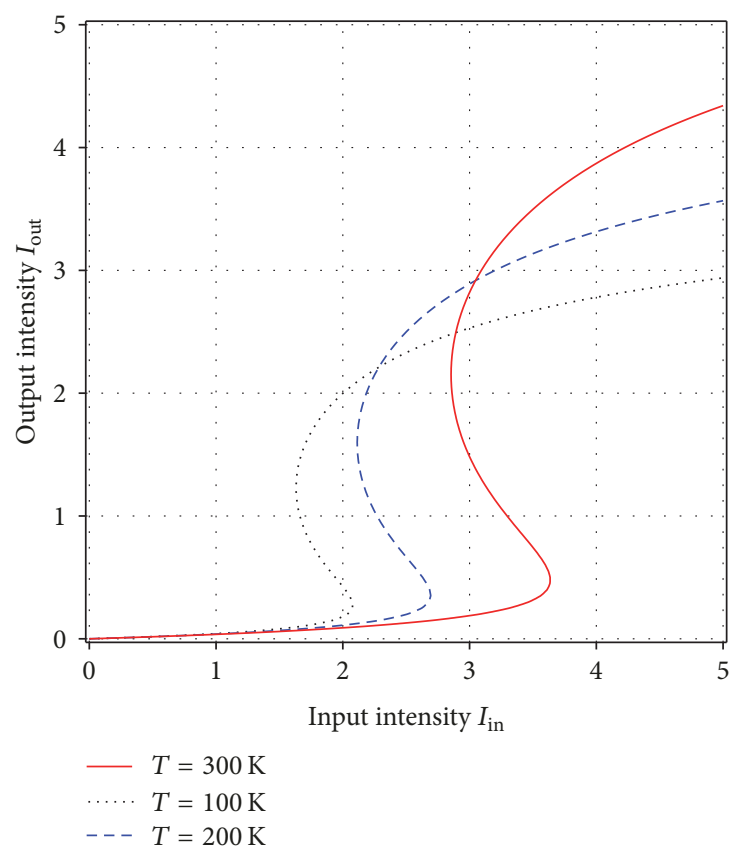

(a)

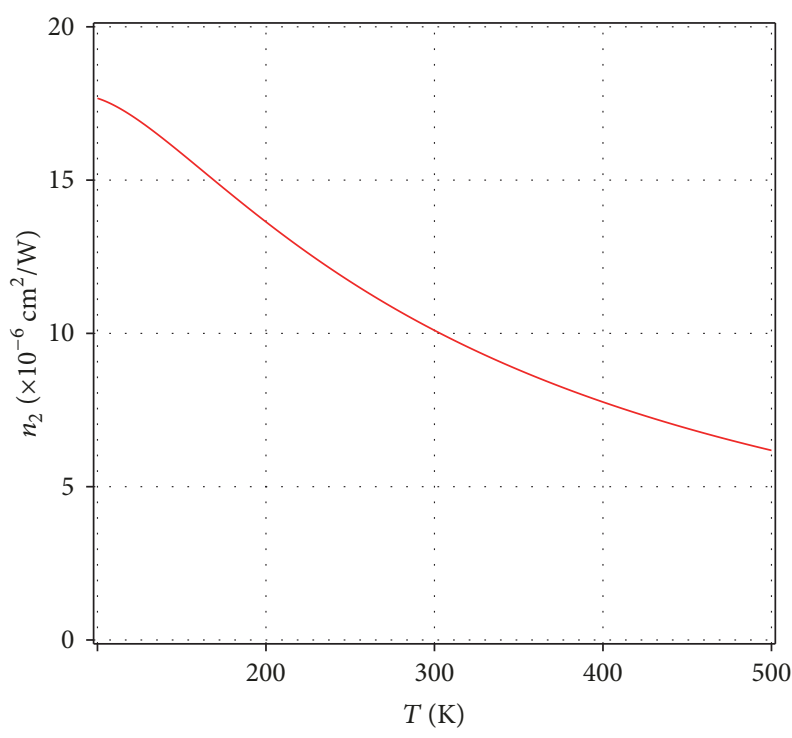

(b)

FIGURE 5: The OB curves at particular values of the temperature (a) and variation of Kerr nonlinear coefficient $n_{2}$ versus the temperature (b). The parameters are $\Delta_{p}=-5 \mathrm{MHz}, \Delta_{c}=0$, and $\Omega_{c}=70 \mathrm{MHz}$.

$8.5 \times 10^{-6} \mathrm{~cm}^{2} / \mathrm{W}$, and $5.9 \times 10^{-6} \mathrm{~cm}^{2} / \mathrm{W}$, respectively. The case of maximum value of the Kerr nonlinearity corresponds to the lowest threshold and width of the OB. Consequently, the threshold and width of the $\mathrm{OB}$ decrease as growing magnitude of Kerr nonlinearity. This behavior is as same as the second case which is indicated in Figure 4. Here, the lowest threshold and width of the $\mathrm{OB}$ are obtained at $\Omega_{c}=60 \mathrm{MHz}$ corresponding to the largest value of the Kerr nonlinearity.

Finally, we studied influence of Doppler broadening by plotting $\mathrm{OB}$ curves at particular values of temperature and plotted variation of the Kerr nonlinearity, as shown in Figure 5. Similar to the cases in Figures 2-4, the results here show that growing temperature leads to reduction of Kerr nonlinearity and thus increases the threshold and width of the OB.

\section{Conclusion}

We have studied the OB behaviors of a unidirectional ring cavity containing an EIT enhanced self-Kerr nonlinear medium under Doppler broadening. In a weak field limit of the probe light, an analytic $\mathrm{OB}$ equation was derived as a function of controllable parameters. It is shown that, owing to the controllable Kerr nonlinearity, the characters of the $\mathrm{OB}$ can be manipulated with intensity and frequency of the coupling field and temperature of the medium. Furthermore, enhancement of the Kerr nonlinearity leads to reduction of the switching intensity thresholds and width of the OB. The analytic model is helpful to understand $\mathrm{OB}$ phenomena using
Kerr nonlinear gaseous material, and it is suitable for finding the related $\mathrm{OB}$ applications at low light intensity.

\section{Conflicts of Interest}

The authors declare that they have no conflicts of interest.

\section{Acknowledgments}

The financial support from the Vietnam Ministry of Science and Technology through the Grant no. ĐTĐLCN.17/17 is acknowledged.

\section{References}

[1] L. A. Lugiato, "Theory of optical bistability," in Progress in Optics, E. Wolf, Ed., vol. 21, pp. 71-216, 1984.

[2] H. M. Gibbs, Optical Bistability: Controlling Light with Light, Academic Press, New York, NY, USA, 1985.

[3] A. Joshi and M. Xiao, Controlling Steady-State and Dynamical Properties of Atomic Optical Bistability, World Scientific Publishing, 2012.

[4] K.-J. Boller, A. Imamolu, and S. E. Harris, "Observation of electromagnetically induced transparency," Physical Review Letters, vol. 66, no. 20, pp. 2593-2596, 1991.

[5] M. Fleischhauer, A. Imamoglu, and P. J. Marangos, "Electromagnetically induced transparency," Reviews of Modern Physics, vol. 77, no. 2, pp. 633-673, 2005.

[6] L. V. Doai, P. V. Trong, D. X. Khoa, and N. H. Bang, "Electromagnetically induced transparency in five-level cascade scheme of 85Rb atoms: an analytical approach," International Journal for Light and Electron Optics, vol. 125, no. 14, pp. 3666-3669, 2014. 
[7] D. X. Khoa, P. V. Trong, L. V. Doai, and N. H. Bang, "Electromagnetically induced transparency in a five-level cascade system under Doppler broadening: an analytical approach," Physica Scripta, vol. 91, no. 3, Article ID 035401, 2016.

[8] D. X. Khoa, L. C. Trung, P. Van Thuan, L. Van Doai, and A. N. H. Bang, "Measurement of dispersive profile of a multiwindow electromagnetically induced transparency spectrum in a Doppler-broadened atomic medium," Journal of the Optical Society of America B: Optical Physics, vol. 34, no. 6, pp. 12551263, 2017.

[9] H. Schmidt and A. Imamoǧlu, "Giant Kerr nonlinearities obtained by electromagnetically induced transparency," Optics Expresss, vol. 21, no. 23, pp. 1936-1938, 1996.

[10] S. E. Harris, "Nonlinear optics at low light levels," Physical Review Letters, vol. 82, no. 23, pp. 4611-4614, 1999.

[11] D. X. Khoa, L. Van Doai, D. H. Son, and N. H. Bang, "Enhancement of self-Kerr nonlinearity via electromagnetically induced transparency in a five-level cascade system: an analytical approach," Journal of the Optical Society of America B: Optical Physics, vol. 31, no. 6, pp. 1330-1334, 2014.

[12] H. Wang, D. Goorskey, and M. Xiao, "Enhanced Kerr nonlinearity via atomic coherence in a three-level atomic system," Physical Review Letters, vol. 87, no. 7, Article ID 073601, p. 073601/4, 2001.

[13] L. V. Doai, D. X. Khoa, and N. H. Bang, "EIT enhanced self-Kerr nonlinearity in the three-level lambda system under Doppler broadening," Physica Scripta, vol. 90, no. 4, Article ID 045502, 2015.

[14] A. Joshi, A. Brown, H. Wang, and M. Xiao, "Controlling optical bistability in a three-level atomic system," Physical Review A: Atomic, Molecular and Optical Physics, vol. 67, no. 4, p. 4, 2003.

[15] Y. Zhang, Z. Wang, Z. Nie et al., "Four-wave mixing dipole soliton in laser-induced atomic gratings," Physical Review Letters, vol. 106, no. 9, Article ID 093904, 2011.

[16] A. Joshi, W. Yang, and M. Xiao, "Effect of quantum interference on optical bistability in the three-level V-type atomic system," Physical Review A: Atomic, Molecular and Optical Physics, vol. 68, no. 1, p. 015806, 2003.

[17] J. Li, "Coherent control of optical bistability in a microwavedriven V-type atomic system," Physica D: Nonlinear Phenomena, vol. 228, no. 2, pp. 148-152, 2007.

[18] Z. Wang, A.-X. Chen, Y. Bai, W.-X. Yang, and R.-K. Lee, "Coherent control of optical bistability in an open $\Lambda$-type threelevel atomic system," Journal of the Optical Society of America B: Optical Physics, vol. 29, no. 10, pp. 2891-2896, 2012.

[19] J.-H. Li, X.-Y. Lü, J.-M. Luo, and Q.-J. Huang, “Optical bistability and multistability via atomic coherence in an $\mathrm{N}$-type atomic medium," Physical Review A: Atomic, Molecular and Optical Physics, vol. 74, no. 3, Article ID 035801, 2006.

[20] X.-Y. Lü, J.-H. Li, J.-B. Liu, and J.-M. Luo, "Optical bistability via quantum interference in a four-level atomic medium," Journal of Physics B: Atomic, Molecular and Optical Physics, vol. 39, no. 24, article 013, pp. 5161-5171, 2006.

[21] M. Sahrai, H. R. Hamedi, and M. Memarzadeh, "Kerr nonlinearity and optical multi-stability in a four-level Y-type atomic system," Journal of Modern Optics, vol. 59, no. 11, pp. 980-987, 2012.

[22] H. R. Hamedi, S. H. Asadpour, M. Sahrai, B. Arzhang, and D. Taherkhani, "Optical bistability and multi-stability in a fourlevel atomic scheme," Optical and Quantum Electronics, vol. 45, no. 3, pp. 295-306, 2013.
[23] J.-H. Li, "Controllable optical bistability in a four-subband semiconductor quantum well system," Physical Review B: Condensed Matter and Materials Physics, vol. 75, no. 15, Article ID 155329, 2007.

[24] L. Ebrahimi Zohravi, R. Doostkam, S. M. Mousavi, and M. Mahmoudi, "Controlling the optical bistability in a kobrak-rice 5-level quantum system," Progress in Electromagnetics Research $M$, vol. 25, pp. 1-11, 2012.

[25] D. X. Khoa, L. V. Doai, L. N. M. Anh et al., "Optical bistability in a five-level cascade EIT medium: an analytical approach," Journal of the Optical Society of America B: Optical Physics, vol. 33, no. 4, pp. 735-740, 2016.

[26] Z. Zhu, A.-X. Chen, Y. Bai, W.-X. Yang, and R.-K. Lee, "Controllable optical steady behavior from nonradiative coherence in $\mathrm{GaAs}$ quantum well driven by a single elliptically polarized field," Modern Physics Letters B, vol. 28, no. 15, Article ID 1450117, 2014.

[27] J. Yuan, W. Feng, P. Li et al., "Controllable vacuum Rabi splitting and optical bistability of multi-wave-mixing signal inside a ring cavity," Physical Review A: Atomic, Molecular and Optical Physics, vol. 86, no. 6, Article ID 063820, 2012.

[28] Z. Zhang, D. Ma, J. Liu et al., "Comparison between optical bistabilities versus power and frequency in a composite cavityatom system," Optics Express, vol. 25, no. 8, pp. 8916-8925, 2017.

[29] Z. Zhang, H. Chen, L. Zhang et al., "Unveiling the relationship between optical bistability and vacuum Rabi splitting," EPL (Europhysics Letters), vol. 117, no. 5, Article ID 53001, 2017.

[30] B. E. A. Saleh and M. C. Teich, Fundamentals of photonics, John Wiley \& Sons, 2nd edition, 2007.

[31] Daniel Adam Steck, “ ${ }^{87}$ Rb D Line Data," http://steck.us/alkalidata. 

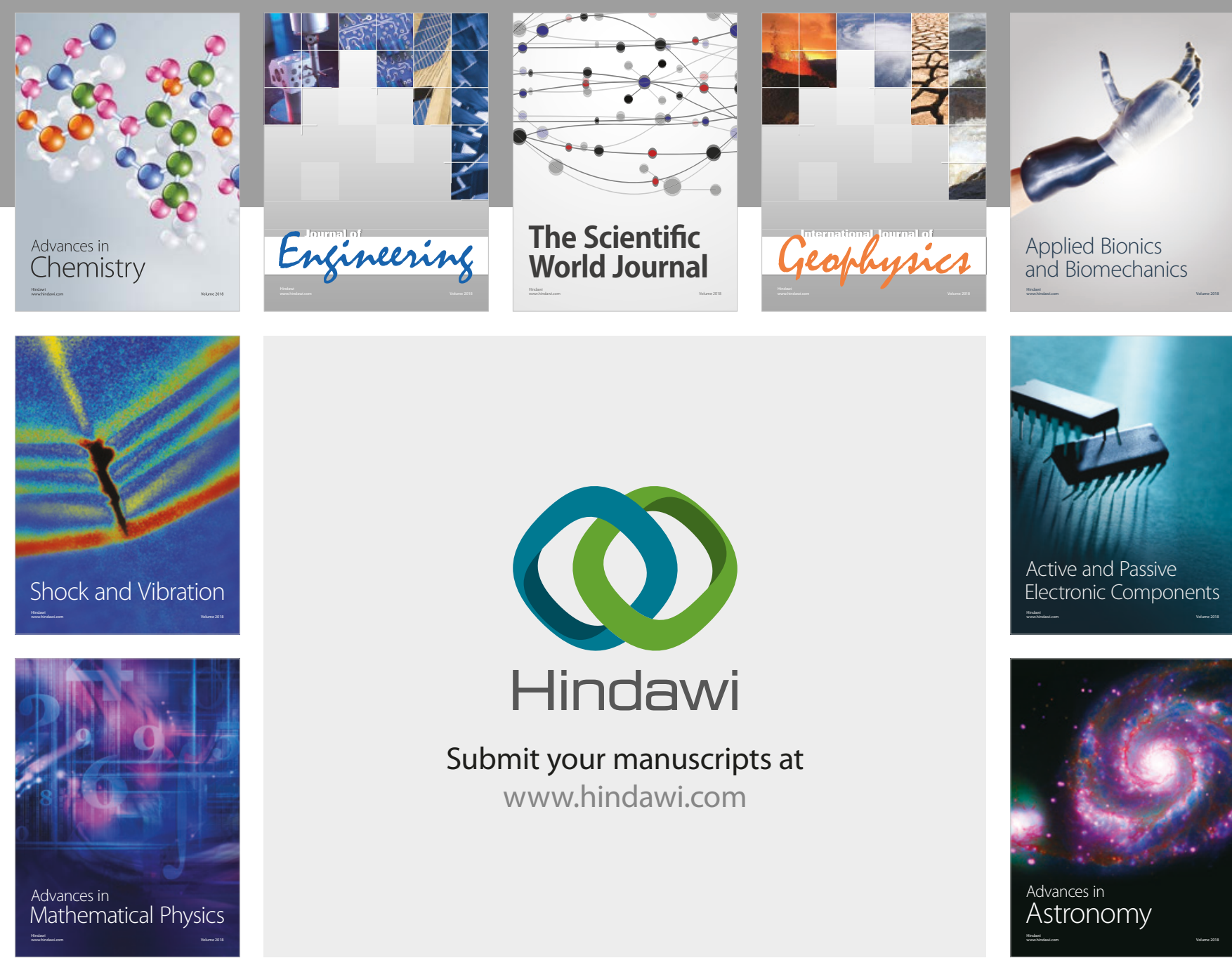

Submit your manuscripts at

www.hindawi.com

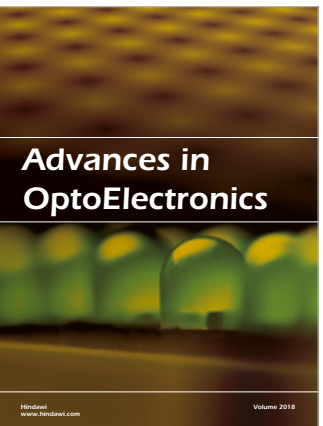

\section{Rotcting Machinery}
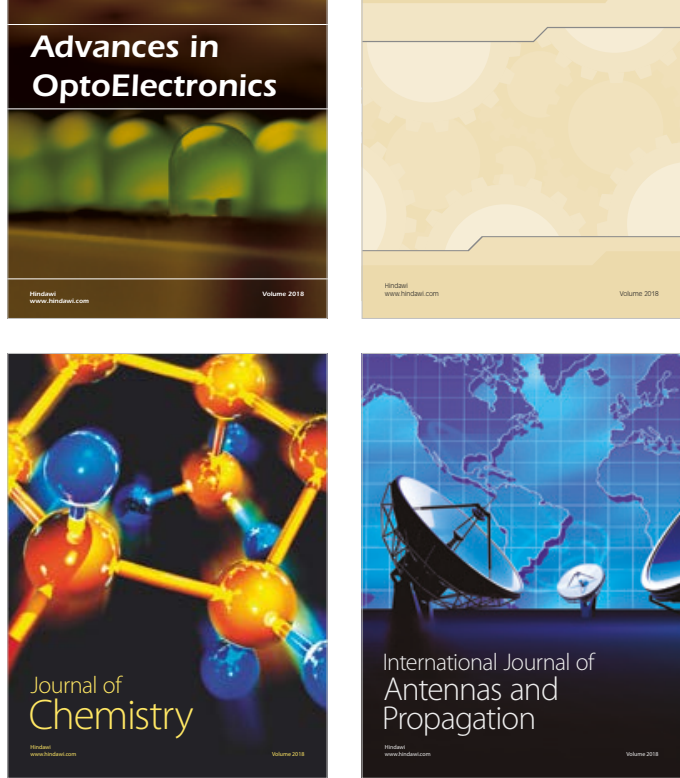

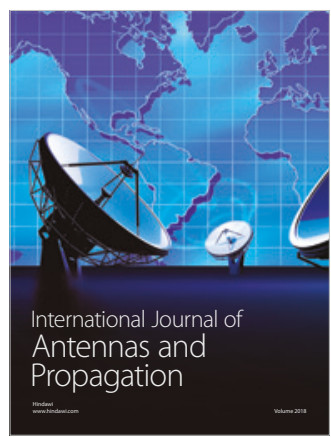

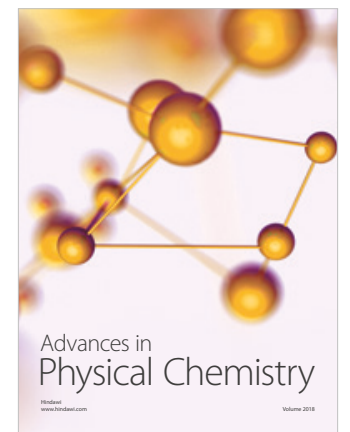

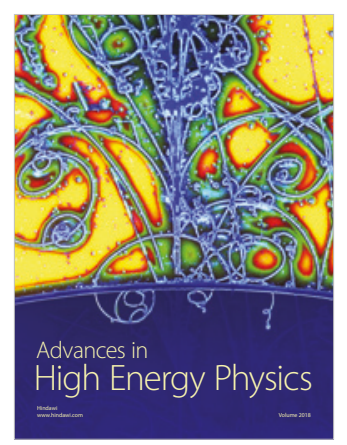

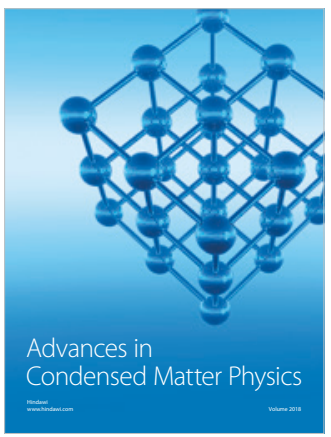

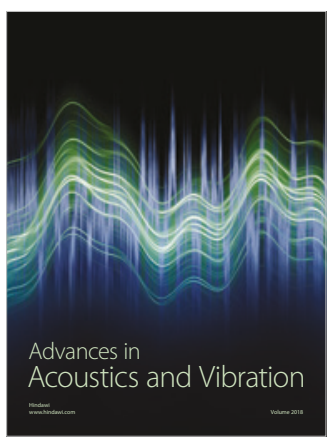

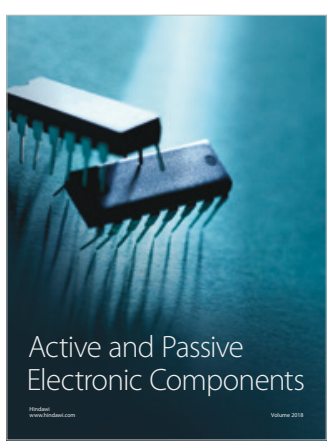
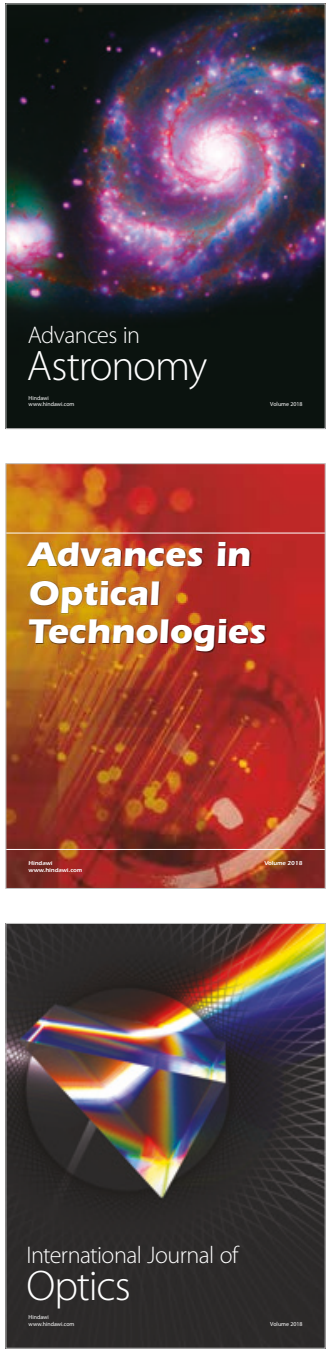